\title{
THE PSI NETWORK MODEL FOR STUDYING DIVERSE COMPLEX DESIGN SCENARIOS
}

\author{
Reich, Yoram (1); Subrahmanian, Eswaran (2) \\ 1: Tel Aviv University; 2: Carnegie Mellon University
}

\begin{abstract}
Design pervades our world in variety and complexity that is difficult to grasp. Different disciplines and researchers take one or few perspectives to study, model, and try to understand design, why it fails and when it succeeds. However, without a comprehensive model, the value of such approaches is limited. We created a framework - the PSI (Problem, Social and Institutional Spaces) framework - that includes several models, ranging from simple to complex, networked, and hierarchical or recursive that can be used to model all design scenarios. In what follows, we demonstrate the network version of this framework, how it is used, and what insight it can present. The diversity of situations we present, mergers and acquisitions of companies, collaborations between industry and university, large multiorganizational projects, and a very complex project, as well as other cases we do not present here, confers the validity of utility and potential of the PSI framework.
\end{abstract}

Keywords: Design management, Design theory, Design methodology

\section{Contact:}

Reich, Yoram

Tel Aviv University

School of Mechanical Engineering

Israel

yoram@eng.tau.ac.il

Cite this article: Reich, Y., Subrahmanian, E. (2019) 'The PSI Network Model for Studying Diverse Complex Design Scenarios', in Proceedings of the 22nd International Conference on Engineering Design (ICED19), Delft, The Netherlands, 5-8 August 2019. DOI:10.1017/dsi.2019.134 


\section{INTRODUCTION}

Design underlies any activity performed by people. From designing meals, careers, and homes, to products, institutions, services, and constitutions; involving a single person to thousands in large organizations, to millions in societies. They may span continents, time, languages and cultures. With such diversity, there have been some efforts in organizational science that focused on micro or macro levels through case studies and other methods (Kozlowski et al, 2000). Nevertheless, the problem of understanding the underlying multi-scale and multi-level nature of design failures and successes require models, frameworks, and tools to collect, analyze and present information about the issues at stake so that designers could synthesize successful solutions (Mihm et al., 2000).

The PSI (Problem, Social and Institutional Spaces) framework is a theory of design and a framework that is intended to model, explain, and address design challenged faced by diverse organizations. In the past, we presented several models of PSI in increased complexity to allow modeling design situations: (1) PSI spaces -3 spaces that need to be aligned; (2) 2-levels PSI - 2 levels of the spaces in which the lower level models the daily operation of the organization and the top models the alignment process; and (3) the PSI matrix, which contains 3 levels of vision/strategy at the top level, reflection, implementation, and monitoring at the middle level, and daily life at the bottom. These models evolved out of the necessity to account for modeling all the aspects of a social entity, be it a small group of designers or a large company. However, real complex design scenarios often involve multiple actors and organizations, each of which has its own PSI model. Capturing the relations between them requires a different interlinked model. The natural progression from the PSI matrix was a network of matrices, where each represents a social entity interacting in a complex endeavor. Here again, the notion of alignment plays a role. We hypothesize that the extension of the basic PSI framework to a network of PSI models provides for explanations and prediction of successes or failures of complex design contexts.

Using a network model is not new in the studies of complex design situations. Network models were used to study distributed work of designers through complex network models (Braha \& Bar-Yam, 2004); to model information flow between designers (Davis et al., 2001) or professionals (Sitton \& Reich, 2018); and used in agent based modeling of human organizational structures, flow simulation and markets (Bonebeue, 2002). Network models of organizations are set up depending on the objective of the model. Therefore, they vary in the details used for modeling (from network topology only to actors with goals and procedures) and the algorithms applied to the network (e.g., algorithms on graphs and simulations of the network operation).

The network model in PSI is a collection of interconnected PSI matrices of organizational units. The matrices are connected together by connecting any one of a matrix cell to a cell in another matrix; for example, people from two companies participate in a common venture will include one matrix for each company and a link between the social space of each to a social space of a third matrix denoting the project. The alignment is exercised also qualitatively here in each matrix and between matrices. This paper presents the extension to the PSI network model using several case studies that show its capabilities and their derived insight. This paper is organized as follows. Section 2 briefly describes the PSI matrix as a framework. Section 3 elaborates the PSI network model and Section 4 provides some examples of its use. Section 5 discusses the study and its consequences and concludes the paper.

\section{AN OVERVIEW OF THE PSI MATRIX}

The PSI framework (Reich \& Subrahmanian, 2015, 2017; Meijer et al., 2014) has been developed as a way to represent the richness of design activities in their context. With it, we have a comprehensive theoretical underpinning of designing (Subrahmanian et al., 2019). To illustrate, consider a design firm, the problem or product space is the framing of the problem and what is being designed; the social space deals with who is involved in the design; and the institutional space specifies how those involved in the design, execute it. These PSI spaces have to be aligned or be in equilibrium for the design work to be successful. For example, improper communication between the partners may lead to designing a poor product. Similarly, if we wish an economic development project to succeed, the local community must be part of the design team and the process should be inclusive and participatory (Reich et al., 1996; Subrahmanian et al., 2017). 
The PSI matrix is the subsequent development of the PSI framework to 3 levels (Reich \& Subrahmanian, 2017) for providing a comprehensive framework to explain the operation of individual social entities in their entirety, see Figure 1. The original framework described above is depicted as the $1 \mathrm{st}$ level in the figure. The 2 nd level is required if the situation goes out of alignment or to stir the organization into new directions. This could happen in many ways, e.g., if the problem turns out to be more complex than envisioned, if the team changes, if its practices no longer fit the situation, if technology changes, or if the company wishes to become a global company. The way to approach such misalignments or new goals is to address these challenges by redesigning the whole PSI at the 1st level. This redesign is performed at the 2 nd level. Consequently, we can consider the 2 nd level as a design activity requiring its own PSI structure. What drives the 2 nd level is a vision $/$ strategy $^{1}$ determined at the top level. The activity of setting the vision is itself a design activity that requires its own PSI. This leads to the matrix in Figure 1.
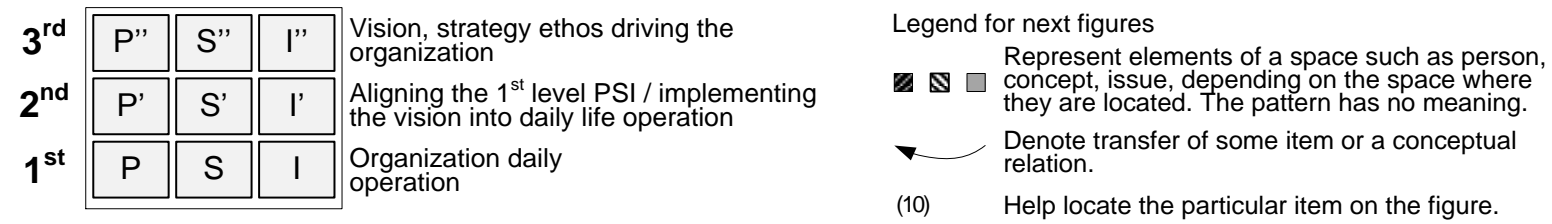

(10) Help locate the particular item on the figure.

\section{Figure 1: The PSI matrix and partial legend for subsequent figures (exchange of people,} information or conceptual relations)

If we use this structure properly, we understand that as the $\mathrm{P}$ in the 2nd level deals with the whole PSI at the 1st level, so should the P at the 3rd level deal with the whole PSI at the 2nd level and below. This means that it is insufficient to determine vision alone in isolation from its implementation, the vision design has to converse with the ability of the organization and other partners to devise a plan to change the organization to where the vision is taking it. However, this plan needs to be implementable by the organization and aligned to its culture, context, problem it addresses and products or their future state. This means that the three levels concurrently evolve through a path that drives the organization towards its vision with some assurance. From this description, we understand that not only do the 3 spaces in each level have to be aligned but that the PSI levels themselves have to be synchronized. Such a conception of an organization resembles an organism living in dynamic equilibrium with its environment and its internal composition and the challenges that both pose for its adaptation to environmental changes. Evolutionary economics, which addresses similar issues, is a study of the evolution of competitive processes through institutional routines and learning, and institutional change (Nelson and Winter, 2002). Their approach is not from a design perspective. Further discussion of a more detailed relationship between that line of work and ours is beyond the scope of the paper.

\section{AN INTRODUCTION TO THE PSI NETWORK MODEL}

We introduce the PSI network model through two merger and acquisition (M\&A) cases. They serve to show that a model of these cases cannot be addressed without its requisite complexity. Consider a thriving, mature company, who has to remain anonymous, in the defense market, whose state could be modelled by an aligned PSI matrix. Every aspect of the organization represented in the matrix works in tandem with others and altogether, vision drives the organization forward. Consider further a startup company, again, whose name we cannot disclose, with its own vision, personnel and work practices who passionately engaged in a civilian disruptive technology to change the future. ${ }^{2}$ The mature company buys the startup and starts to merge its assets into itself. It takes short time before many of the startup employees leave the company. There is no consideration of the cultures of the organizations (arrow (1) in Figure 2(a)), one quite rigid (I in B) and the other much less structured (I in A). There is no synchronization of their visions (arrow (2), P' 'in both A and B), or the aspiration of

\footnotetext{
${ }^{1}$ Vision and strategy are used interchangeably.

${ }^{2}$ Strategy of exploration (discovery) versus exploitation (maximizing efficiency) require different organizational structures to execute them (Lewin, 1999). To move from exploitative mode to translation of exploration that is absorbed in the organization requires organizational restructuring (Lewin, 1999).
} 
the startup employees. There was an assimilation of the startup's products (arrow (3) moving all the $\mathrm{P}$ of A - patterned square - into the products of B - now represented as a small patterned square). Also all the workers of the startup (patterned S of A) join (arrow (4)) into the workforce of B (small patterned square in $\mathrm{S}$ of $\mathrm{B}$ ). These moves are done independently without aligning the organization. We came to know about this case at the beginning of the process and predicted its failure. In reality, sometime later, the result of the acquisition was a failure, ending with only the mature company as the products of the startup dissolved and most of its workforce left company B.

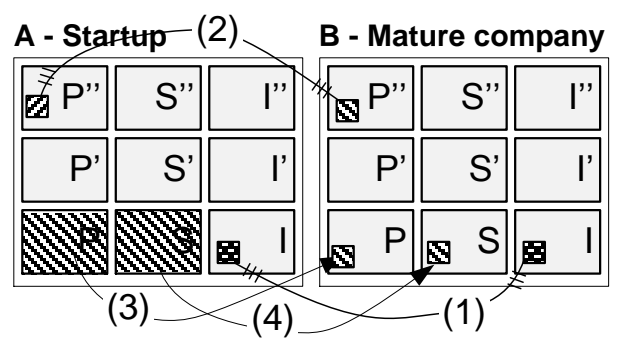

(a) Failed acquisition

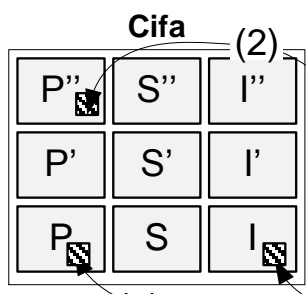

(3)

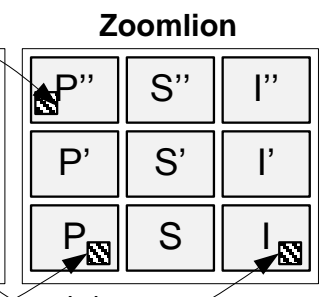

(1)

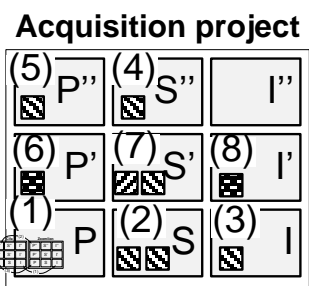

(b) Successful acquisition

Figure 2: PSI network model of two M\&A projects

Another completely different acquisition is manifested in the acquisition of the Italian company Cifa by the Chinese company Zoomlion, Figure 2(b). In this case, the acquisition materialized after making sure that the cultures (1) and visions (2) of the two companies are aligned (bi-directional arrows), even though not identical. The acquired company continued to carry its brand, management, and heritage. In fact, 5 years after the acquisition, it founded a museum to commemorate its history and achievements. Consequently, the combined company enjoys diversity of ideas and continues innovating its products in both locations (3). We described two very different approaches to M\&As but their essence is not described with the models displayed in (a) or (b). What these models lack is an explicit account of the act of acquisition.

We can model this act in at least two ways. First, in the case of company B acquiring A, we could place the activity at the 2nd level PSI of B. Part of the vision of B, to grow it ( $\mathrm{P}$ ') was not aligned with the actual practice (P, S, I). Aligning it in P', required expanding $\mathrm{P}, \mathrm{S}$ and potentially modifying I. The acquisition ended with a non-integrated $\mathrm{P}$ and $\mathrm{S}$ that grew at the moment of acquisition but shrank due to lack of employee retention (social space), which was not even considered as part of the acquisition.

A second approach is to create a separate PSI matrix for the acquisition project depicted in (b), shown in Figure 2(c). We note that the problem space $\mathrm{P}$ of this matrix is the acquisition project, represented as a shrunk picture of Figure 2(b) - (1). Representatives from both companies (2, patterned squares) explored the potential of acquisition (1) in an open dialogue (3, patterned square). This approach was driven by the managers' (4) vision (5) respecting heritage, culture, innovation, and cooperation. Such vision for the acquisition project, permitted keeping the two entities separated after the acquisition, with their particular cultures and managerial approaches (I) in (b). Using a separate PSI matrix for the acquisition project allows for much greater fidelity of modeling. For example, it makes explicit the fact that this project is a separate entity that has to be managed and also improved. In the case of Zoomlion and Cifa, since the two brands remained intact after the acquisition, their work had to be coordinated continually - a task that could be assigned to the same acquisition matrix and monitored all the time by a 2 nd level PSI - P'S'I' $(6,7,8)$. This makes sure that the project itself practices constant reflection and has its own vision and objectives (5) that could be different from any of the companies. Similarly, we could envision any challenge in any existing PSI as receiving its own matrix. The dilemma of whether to approach it in this way is whether the extra attention reflects the challenge in complexity, following the law of requisite variety (Ashby, 1958).

\section{FURTHER EXAMPLES OF USING THE PSI NETWORK MODEL}

\subsection{Vision as a driving force of projects - La Défense Metro Station Project}

This case describes a large scale project, developing the metro station in the La Défense district, Paris; it was constructed by interviews with George Amar and verified by him. This case demonstrates how 
several organizations, each with its own PSI, collaborated together in a time-limited project, which has its own PSI (as in Figure 2(c)); this allows inspecting what happens when the project ends and its PSI matrix vanishes. Further, the case shows how interaction between these spaces is done at the levels of cells in the matrix.

RATP was in charge of the project. One day, RATP to-be CEO (1) invited Georges Amar (GA) (2) to discuss the project (3). Modeled in the RATP matrix, it would be located at the day-to-day operation. However, if we wanted to describe it more precisely, we would need to form an initial PSI matrix template for the project (4) and place them in S". They are discussing the nature or vision of the project ( $\mathrm{P}$ ", 5). He (1') proposed that GA (2') would be the leader of the project knowing him for his abstract conceptual way of thinking (6, a dimension of $\mathrm{S})$ and as an expert in mobility (alignment between knowledge required in P (7) and people in S). GA asked to consider the offer for several days and returned with a 10-page document explaining his conception (5) of the metro station. GA changed the language of discussing the project from transportation, focusing on hardware and its needs and constraints, to the language of the station and its environment as a living system. In a living system, the station is the organ that brings life, blood; it becomes the heart. This concept was based on years of research and study on the importance of the nodes in transportation network rather than the links - a conception that challenged the common transportation paradigm. The CEO agreed and GA took the project (negotiation at the I" (8) between the two participants at the S'). This concept became part of the vision of the project (P').

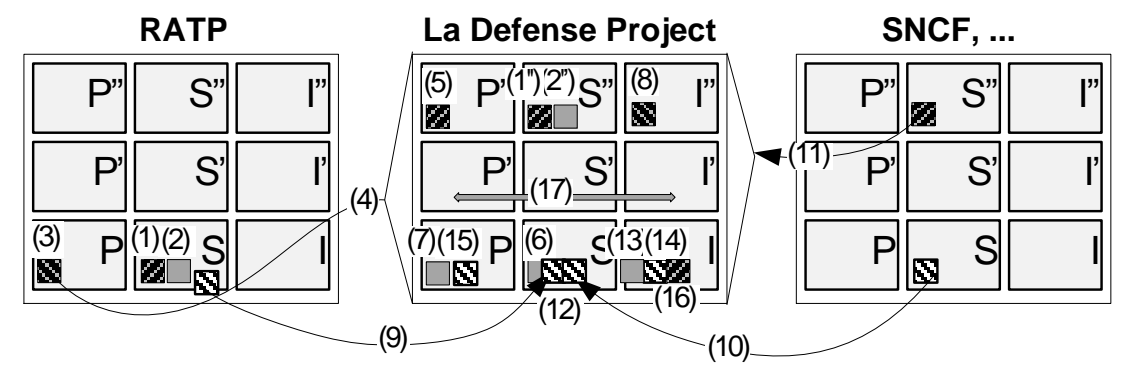

Figure 3: PSI network model of The La Defense Metro Station project

Initially, the project was without serious budget but still, GA had his own ideas about building the project team $(\mathrm{S}, 6)$. He wanted a person with a perspective of the district; he got a retired driver (9) from the company who was supposed to provide the sanity check of the different ideas. It turned out later that the driver's view was so critical that he contributed to the concept also in spite of him not being a professional engineer. GA brought into the project (10) few more people from each of the participating transportation organizations such as Metro-bus and SNCF (French Rail).

GA enlisted people (10) into the project and executives (11) to support it by presenting them with the concept (5) and suggesting that he could not do the project without them. This effectively presented them the misalignment problem between the vision (5) the knowledge required (7) and the social space S (12); GA was asking for their small contribution in aligning the situation. Partners such as SCNF agreed to come on board and sent a representative and so did others (10). GA relayed the information about such contributions of other partners to RATP CEO (1, 1") to obtain additional support from him. GA did not assume the position of know-all but that of a facilitator asking for true participation (13) of other necessary partners. It was a position of a student and not of a chief. GA obtained office location at the site to enable moving the whole team to a single location (14) thus releasing them from their local organizational culture and allowing them to form a coherent team and build a coherent culture. The team allowed addressing the whole project rather than working on it as a collection of independent projects, executed each at a partnering organization that could never form the true whole (see Boeing Dreamliner case later).

Questions that needed creative solutions or conceptualizations were for example (15): designing a clear maze. Transportation network tends to develop into a maze, so how could a maze be clear, an antithetical construction. Other ideas were no glass for protecting the employees in the information center, or combined information booth at the center of the station. The creative process followed critiquing the existing model and detecting a new paradigm that would inspire further elaboration (16). This project presents an example how vision (5) could drive diverse people (12) into a major achievement and innovation, motivating participants to leave behind their past cultural habits and take part in a paradigm-shifting project. 
Among other things, we see in the project a missing explicit working 2nd level (17). We also do not see explicit participation of executives, no links between the different $\mathrm{P}$ " in the model and no attempt to influence the vision of the participating organizations; there was no particular project aiming to assimilate the project values back into the partner organizations. Consequently, any change in the project structure may eliminate some of its achievements and it might not translate to future projects. Indeed, at the end of the design phase of the project, GA left and another person became the construction project manager. The project vision did not stick completely in the construction. Some elements were removed from the design.

Once the project ended, RATP wanted to replicate the model in many other locations. However, without intimate involvement in the location with the help of local people, such project would not work; its S would not be aligned with its $\mathrm{P}$. Consequently, there should be a separate project for each location and not mass production of stations. Further, as predicted by the model, the ideas developed through this project (4) and the experience implementing them did not influence the partner organizations as it could have. No lasting effect was noticed. It lived temporarily in the project due to the particular team makeup and its leader.

In this case, the network model is necessary to describe faithfully the project evolution and the fate of its vision than with a single matrix. A sequence of matrices, each for different stages in the project lifecycle would have provided an even better description.

\subsection{A project as a place for incubating a community - $\mathrm{n}$-dim}

$\mathrm{n}$-dim is a name that referred to a project at the Engineering Design Research Center (EDRC) at Carnegie Mellon University (CMU) but also to a group of people or even a dynamic community whose makeup changed dramatically over the years with a flux of people coming in and out of the group (Subrahmanian et al., 1997). The group of the n-dim project participated in various projects in academia and industry. $\mathrm{n}$-dim is used here as a community or an organization engaged in projects.

The project started when a group of people from Westinghouse (1 in Figure 4) approached people at CMU (2) to collaborate on supporting engineering design. Westinghouse Corporation was one of the largest American corporation in the electrical, nuclear and transportation businesses for 100 years before it was dismantled. They were the pioneers of radio broadcasting, AC electric power and driverless metro trains and a major American competitor of GE. CMU people worked at an environment whose worldview (3) was that of individual information processing model of cognition to replicate human designers as expert systems, following the leadership of Herbert Simon and Allan Newell (4). Trying practices (5) and solution approaches (6) derived from this vision, was not the focus of the Westinghouse team; they came to the project with a different vision (7) altogether that was not aligned with (5) or (6). While the vision was not fully articulated, its manifestation in the research process was (8): asking us (9), also from the EDRC, to partner with several of their researchers to learn how a "group of engineers" work, by working with, and observing with them, the processes they use. Subsequently Westinghouse brought cognitive engineers and nuclear engineers to the team (more of 1) to form a joint participatory team to move to their vision of engineering design (7). The discussion (10) between Westinghouse (11) and us (9) really started at the 2 nd level of the project regarding its research methodology (12), understanding that only this approach would lead to uncovering issues that will then be addressed as research projects at the 1st level (13). This involved participatory designing a support for the engineering process (14), with a team of engineers (effectively new participants added to the project team) (15) working in the context of creating a control system for nuclear power plants that included new distributed computational technology (16).

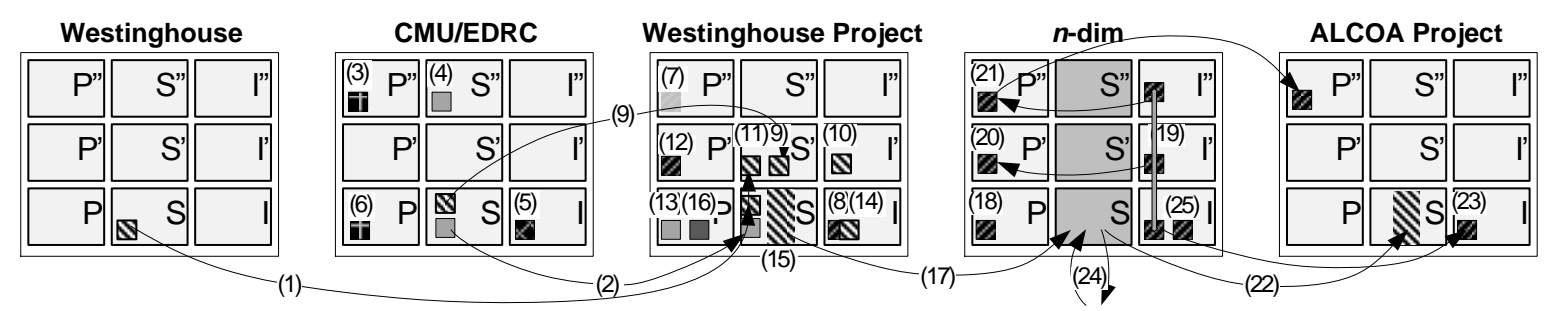

Figure 4: PSI matrixes of the $n$-dim project

We note that the top level PSI was not explicit in the PSI project matrix; and even the 2nd level was not necessarily continuing its operation as the project unfolded. As such, the project was not 
sustainable. Nevertheless, as the project continued, the n-dim group crystalized (17) and begun to create its own identity. This was reflected by a focus on information management in design (18) and creating a worldview of participation, attitude to learn with industry to address real problems, without presuppositions or ego involved and respect to all participants coming from diverse perspectives (19). This external attitude was equally reflected inside as the mode of operation of the group: no hierarchy, mutual respect, learning attitude and concern to real practice as the criterion for quality research - a clear instance of "practice what you preach" (Reich, 2017). The group was crystalizing in a bottom-up fashion, using the same worldview to create its reflexive mechanisms (20) and ethos (21), gradually making explicit all the cells in the PSI matrix, while making sure they are aligned.

Similar approach, now embedding the emerging group methodology and ethos, was exercised when working on a project on integrating materials databases across ALCOA research Labs. Here the group was asked to join the project (22) to replace other researchers who had a narrow, purely technical perspective prevalent at CMU. The team brought its methodology (23) and ethos (21) to the project which was adopted by all project participants. By this time, we were able to articulate our ethos as a model of extended participation in design (Reich et al., 1996). From this point on, our engagement always went with understanding that the success of defining the project and implementing it requires a reflexive, inclusive and mutually learning open environment (evolution of (19)). The ethos was translated into an agreed upon model of collaboration (part of the I' and I spaces of all future projects). Similarly, the sharing of the intellectual property was agreed upon, so that both sides could preserve their identity (also parts of I' and I; similar to the Zoomlion and Cifa case) and still produce results that are useful to the daily operations of the industrial partners; i.e., that the project has a lasting effect on all participants (in contrast to the La Defense project).

Over the years, the n-dim worldview (19) and ethos (21) further crystalized, creating a well aligned flexible group. They were reflected on the group operation in several ways. First, it was easy for students and other people to come into the project and quickly assimilate and find their respectful place in different projects as regular contributors (24). A few who could not accept the vision left very shortly after joining the group. It is fair to say that many members of the group still share the ethos today in their careers. Second, the ethos evolved the group practices by directing building a computational infrastructure, also called n-dim (25) that would support the ethos (Subrahmanian et al., 1997; Reich et al, 1999).

This case shows that in contrast to the La Défense example, and similar to the Zoomlion and Cifa acquisition, a strongly aligned project, could have lasting effects on its members as well as could lead to the formation of a dynamic community.

\subsection{Aligning PSI network - Boeing Dreamliner Project}

This example is only described briefly for lack of space. It is interesting in several aspects, from which we mention only two. First, in spite of its complexity, when it was initially presented (Reich and Subrahmanian, 2015), we used only a 2-level PSI framework that focused on Boeing use of extensive outsourcing in the project while laying off personnel needed for the subsequent integration of components. This created misalignment between the problem $(\mathrm{P})$ and the skills available (S). To better understand the context and the resolution of the problem, a network model was used that contains part of the information available, see Figure 5 (Smulders et al., 2018). Due to its complexity, there is a timeline attached to the model. The model starts with around the time of McDonnell Douglas merger with Boeing in 1997 (1). We did not collect enough information and did not intend to model this merger with its own PSI matrix to better understand it. Consequently, while we know that the employees of both companies became employees of the merged company, we do not know how the practices, culture, or ethos of the new company evolved and if it was shared by all employees.

The project (787 (2003) matrix) was initiated with misaligned (2) decision at the P" (3) and I (4) of the project without thinking about P' or listening to potential pitfalls in the model (5) analyzed by an expert in the $S^{\prime}$ at Boeing (6). There was no proper setup of 2 nd level PSI in this project (7, even if existed, it did not include participants from all project partners like in the Westinghouse project). All these were in addition to the misaligned 1st level PSI from extensive outsourcing and personnel layoffs (8). This completely misaligned PSI matrix was therefore destined to fail as it did. There was also misalignment between the project and most partners (9) whose practices were insufficient for a project like this. Boeing tried numerous ways to resolve the situations, but without directly fixing the misalignments, these efforts failed. At some point, realizing it requires a surgical solution, Boeing 
quickly performed steps that clearly aligned the matrix (10), allowing the project to get back on track (787 (2008)). The following aligning activities were performed: (a) hiring back people with integration skills (11) - aligning the integration knowledge required in $\mathrm{P}$ with skills at $\mathrm{S}$; (b) establishing an integration facility to align the process with the apparent complexity of the product ((12) in I); (c) sending engineers to other subcontractors facilities to monitor their operation and quality (13, establishing or improving subcontractors S', aligning subcontractors matrices); and (d) buying subcontractors that could not deliver quality products on their own even with Boeing engineers on their site (14, whose PSI matrices could not be aligned with the project $)^{3}$. All these activities could have been recommended by the PSI network model, their totality was needed to resolve the situation and no other prior attempt by Boeing succeeded without alignment. The initial failure of the project due to misalignment of the PSI network and its subsequent resolution by aligning the PSI network makes the Dreamliner case a validation of the PSI as a theory of design. Further refinement can be done to this model to better understand how exactly Boeing got to this situation, in spite that it had the knowledge $(5,6)$, and why it took it a while to understand and resolve, but that is beyond the scope and space of this paper.

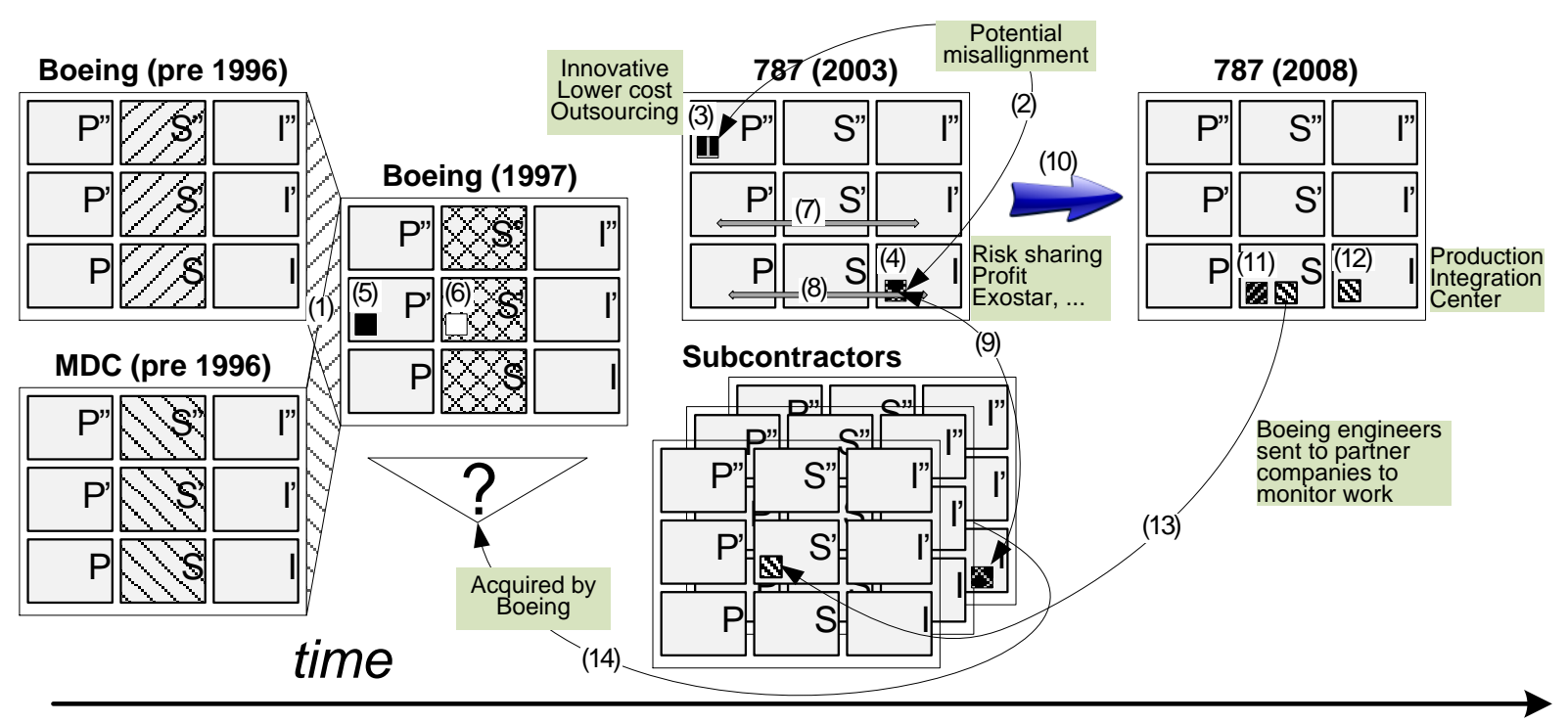

Figure 5: Boeing Dreamliner's project

\section{DISCUSSION AND SUMMARY}

Table 1 summarizes the five case studies, demonstrating a variety from simple to complex network models. We have seen that misalignment can occur in many ways within and between matrices of a network and fixing it may require diverse means as in the Dreamliner project. Not all situation details can be captured by the PSI network, which is qualitative. Nevertheless, it can be augmented by additional models for detecting misalignments and aligning them as in (Bekius and Meijer, 2018). Furthermore, it can be augmented with quantitative models such as DSM (design structure matrix) for attempting alignment between, e.g., the product and institutional spaces (Colfer and Baldwin, 2016).

The PSI network model is a natural progression of the PSI framework expanded to model complex design situations. The network is hierarchical in the sense that we can zoom into a PSI matrix (e.g., early Dreamliner model) and model it with a network or zoom into an action (e.g., acquisition of subcontractors in the Dreamliner project) in any PSI model and model it with a matrix or a network. This allows for significant flexibility in spending modeling effort where necessary. Similarly, aligning between an organization and its project vision or some other objective may require a separate project for aligning because new projects or new architectural configuration of products will lead to new PSI.

\footnotetext{
${ }^{3}$ Recall the first M\&A case and its associated issues and failure. This move should have been done carefully to make sure that following the acquisition all assets: facilities and people remain available to Boeing. Understanding these acquisitions better and making sure they succeed would require their own PSI matrix.
} 
Table 1: Summary of 5 real PSI network cases. The data column notes whether the authors were involved or had direct access to participants in the case (inside) or whether it relies on public information.

\begin{tabular}{|c|c|c|c|c|}
\hline Type & PSI model & Data & Organizations & Comments \\
\hline \begin{tabular}{|l|l|}
1 & M\&A 1
\end{tabular} & $\begin{array}{l}\text { "matrix", } \\
\text { network }\end{array}$ & Inside & $A \& B$ & $\begin{array}{l}\text { Failure due to misalignment of cultures and not } \\
\text { forming a project PSI }\end{array}$ \\
\hline 2 M\&A 2 & network & Public & Zoomlion \& Cifa & $\begin{array}{l}\text { Success due to an aligned acquisition matrix, } \\
\text { aligned with culture and vision of organizations }\end{array}$ \\
\hline $3 \begin{array}{l}\text { La } \\
\text { Défense }\end{array}$ & $\begin{array}{l}\text { "matrix", } \\
\text { network }\end{array}$ & Inside & $\begin{array}{l}\text { RATP, SNCF, La } \\
\text { Défense district, .. }\end{array}$ & $\begin{array}{l}\text { Aligned project with limited life span; } \\
\text { alignment depends heavily on people } \\
\text { participating in the project without sustainability } \\
\text { mechanisms }\end{array}$ \\
\hline n-dim & network & Inside & $\begin{array}{l}\text { Westinghouse, } \\
\text { CMU/ EDRC, n- } \\
\operatorname{dim}, \text { ALCOA, ... }\end{array}$ & $\begin{array}{l}\text { Project turning to a long lasting community with } \\
\text { strong vision driving it }\end{array}$ \\
\hline Dreamliner & $\begin{array}{l}\text { 2-level, } \\
\text { network }\end{array}$ & Public & $\begin{array}{l}\text { Boeing, many } \\
\text { suppliers }\end{array}$ & $\begin{array}{l}\text { Intricate operation that is seen simple through } \\
\text { the PSI network lens; validation of the PSI } \\
\text { theory }\end{array}$ \\
\hline
\end{tabular}

The network model aids the use of the time dimension, reflecting organizations evolution and the influence of projects on organizations (e.g., n-dim and Dreamliner). In the future, this will ease the finding of patterns of PSI matrices interactions, misalignments and successes or failure of projects or organizations at different levels of the problem. To illustrate how patterns can emerge and be reused, consider the first M\&A case and the acquisition of subcontractors in the Dreamliner case, which may be considered as duplicates of the first M\&A case. Understanding the issues in the M\&A case will lead us to much better handing of the acquisition in the Dreamliner case. Finding patterns in PSI networks remain future work.

Only the network model allows us to reflect on the relationships between organizations and their projects including the evolution of projects into organizations (e.g., n-dim case) and vice versa (e.g., in the case of M\&A). Organizations or communities operate extensively through projects. Whenever an organization seeks to achieve a significant objective, it will setup a project with personnel, resources, rules and objectives to execute it. An organization will run numerous projects simultaneously and some with different partners - organizations and projects have many to many relations.

A clear issue that comes to mind is the relation between the organizational ethos or vision and the vision of each of its projects. Can a person or any social entity participate in many social setups with differing ethos without difficulty? For example, RATP would have to be involved in any transportation project around Paris and the new project would have to resolve its ethos with that of the company. In contrast, n-dim could have chosen to participate only in projects whose projected or incubated ethos matched its own. Managing such relationship becomes difficult if organization ethos and one of its project ethos differ significantly, as members of the project are also members of the organization. Reflective organizations must be aware of such issues and resolve them.

While the PSI framework seems extremely useful, it is not trivial to create PSI models. There could be multiple ways to interpret information (e.g., is it a daily life activity or reflection at the 2 nd level) and use different levels of granularity in modeling. We foresee that as experience is gathered and patterns are formed, that the creation of models would become easier.

Last, while presently, we have no algorithmic support for the network model like in social networks, we foresee that we use it as a framework with all the other network models for representing detailed phenomena. Some developments along these lines are underway.

The PSI network is not only a drawing tool. There is insight gained from studying numerous PSI analyses and extracting patterns. For example, the need for a strong 2nd level PSI emerged from many PSI analyses of successful and failed projects. From PSI, it is clear that projects without such level (M\&A1, La Defense, and initial Dreamliner) would fail or would not have lasting effect while those with it (M\&A2, Westinghouse project, and Dreamliner after alignment) have a chance to succeed. Without the language of PSI, it would have been difficult to distill this pattern and use it.

We introduced a new type of PSI model - the network. It allows us to model complex design situations. We gave five real examples of its use and the insight that could be derived for design 
practice by using it. The way to move fast forward in further developing and using the PSI framework is through partnerships with different people and groups. We are looking forward to using PSI to help us form such successful partnerships to expand its scope of application.

\section{REFERENCES}

Allen, T.J. (1984), Managing the Flow of Technology: Technology Transfer and the Dissemination of Technological Information within the $R \& D$ Organization. MIT Press Books.

Ashby, W.R. (1958), "Requisite variety and its implications for the control of complex systems", Cybernetica, Vol. 1, pp. 83-99.

Bekius, F. and Meijer, S. (2018), "The redesign process of the timetable for the Dutch railway sector: a theoretical approach", International Journal of System of Systems Engineering, Vol. 8 No. 4, pp. 330-345.

Braha, D. and Bar-Yam, Y. (2004), "Topology of large-scale engineering problem-solving networks", Physical Review E, Vol. 69 No. 1, p. 016113.

Bonebeau, E. (2002), "Agent based modeling: Methods and techniques for simulating human systems", Proceedings of the National Academy of Sciences, Vol. 99 No. 3, pp. 7280-7287.

Colfer, L.J. and Baldwin, C.Y. (2016), "The mirroring hypothesis: theory, evidence, and exceptions", Industrial and Corporate Change, Vol. 25 No. 5, pp. 709-738.

Davis, J.G., Subrahmanian, E., Konda, S., Granger, H., Collins, M. and Westerberg, A.W. (2001), "Creating shared information spaces to support collaborative design work", Information Systems Frontiers, Vol. 3 No. 3, pp. 377-392.

Kozlowski, S.W.J. and Klein, K.J. (2000), "A Multilevel Approach to Theory and Research in Organizations: Contextual, Temporal, and Emergent Processes”, In: Klein, K.J. and Kozlowski, S.W.J. (Eds.), Multilevel Theory, Research and Methods in Organizations: Foundations, Extensions, and New Directions (pp. 390). Jossey-Bass, San Francisco, CA

Lewin, A.Y., Long, C.P. and Carroll, T.N. (1999), "The coevolution of new organizational forms", Organization Science, Vol. 10 No. 5, pp. 535-550.

Mihm, J. and Sosa, M.E. (2007), "Organization design for new product development", in Handbook of New Product Development Management, Routledge, pp. 181-214.

Meijer, S., Reich, Y. and Subrahmanian, E. (2014), "The future of gaming for design of complex systems", Back to the Future of Gaming, pp.154-167.

Nelson, R.R. and Winter, S.G. (2002), "Evolutionary theorizing in economics", Journal of Economic Perspectives, Vol. 16 No. 2, pp. 23-46.

Reich, Y. (2017), "The principle of reflexive practice", Design Science.

Reich, Y., Konda, S.L., Monarch, I.A., Levy, S.N. and Subrahmanian, E. (1996), "Varieties and issues of participation and design”, Design Studies, Vol. 17 No. 2, pp. 165-180.

Reich, Y., Konda, S., Subrahmanian, E., Cunningham, D., Dutoit, A., Patrick, R., Thomas, M. and Westerberg, A.W. (1999), "Building agility for developing agile design information systems", Research in Engineering Design, Vol. 11 No. 2, pp. 67-83.

Reich, Y. and Subrahmanian, E. (2015), "Designing PSI: an introduction to the PSI framework", In Proceedings of the 20th International Conference on Engineering Design (ICED 15), Milan, Italy, 7.15.

Reich, Y. and Subrahmanian, E. (2017), "The PSI matrix-A framework and a theory of design", In Proceedings of the 21 st International Conference on Engineering Design (ICED 17), Vancouver, Canada, 21-25.08. 2017.

Sitton, M. and Reich, Y. (2018), "EPIC framework for enterprise processes integrative collaboration”, Systems Engineering, Vol. 21 No. 1, pp. 30-46.

Smulders F., Reich Y. and Subrahmanian E. (2018), "The Dreamliner's bumpy road to takeoff. Could Boeing have known?", 11th Design Theory SIG Workshop, Paris, 30-31 January 2018

Subrahmanian, E., Reich, Y., Konda, S.L., Dutoit, A., Cunningham, D., Patrick, R., Thomas, M. and Westerberg, A.W. (1997), "September. The N-Dim approach to creating design support systems", In Proc. of ASME Design Technical Conf.

Subrahmanian, E., Eckert, C., McMahon, C., and Reich, Y. (2017), "Economic development as design: Insight and guidance through the PSI framework", In Proceedings of the 21st International Conference on Engineering Design (ICED 17), Vancouver, Canada, 21-25.08. (pp. 229-238).

Subrahmanian, E., Reich, Y. and Krishnan, S. (2019), We the Designers, MIT Press. 